\title{
UNBOUNDEDNESS OF FIBER INVARIANTS OF CANONICALLY FIBRED VARIETIES OF GENERAL TYPE
}

\author{
MENG CHEN AND ZHI JIANG
}

\begin{abstract}
We answer an open question concerning the boundedness of canonical fiber spaces in high dimensions and prove the following: for any set of integers $n \geq 3,0<d<n$ and $N>0$, there exists a nonsingular projective $n$-fold $X$ of general type so that $X$ is canonically fibred by $d$-dimensional varieties $F$ with $p_{g}(F) \geq N$. This disproves the desired boundedness parallel to Beauville's boundedness theorem (Bea $)$ in the surface case.
\end{abstract}

\section{Introduction}

We work over any algebraically closed field $k$ of characterictic 0 (for instance, $k=\mathbb{C}$ ). In this article, we assume that $X$ is a minimal projective variety of general type, namely the canonical (Weil) divisor $K_{X}$ is nef and big and $X$ has at worst $\mathbb{Q}$-factorial terminal singularities. We call the integer $p_{g}(X):=\operatorname{dim}_{k} H^{0}\left(X, K_{X}\right)$ the geometric genus of $X$. Denote by $\left|K_{X}\right|$ the canonical linear system of $X$. The smallest positive integer $r_{X}$ such that $r_{X} K_{X}$ is Cartier is called the Cartier index of $X$. When $r_{X}=1$, we say that $X$ is Gorenstein.

Assume $p_{g}(X) \geq 2$, the global sections of $\mathscr{O}_{X}\left(K_{X}\right)$ defines a map

$$
\varphi_{1}:=\Phi_{\left|K_{X}\right|}: X \rightarrow \mathbb{P}^{p_{g}(X)-1},
$$

which is called the canonical map of $X$. The rational map $\varphi_{1}$ belongs to two types: (I) $\varphi_{1}$ is generically finite; (II) $\operatorname{dim} \overline{\varphi_{1}(X)}<\operatorname{dim} X$, in which case we say that $X$ is canonically fibred by d-dimensional varieties, where $d:=\operatorname{dim} X-\operatorname{dim} \overline{\varphi_{1}(X)}$.

When $n=1$, it is well-known that $\varphi_{1}$ is either birational or a double covering.

When $n=2$, it is clear from the surface theory (see, for instance, [Bea, BHPV]) that either $\varphi_{1}$ is generically finite of degree $\leq 36$ or $X$ is canonically fibred by curves of genus $\leq 36$.

2010 Mathematics Subject Classification. 14E05, 14J40.

Key words and phrases. Canonical maps, geometric genus, varieties of general type.

The first author was supported by National Natural Science Foundation of China (\#11231003, \#11171068, \#11121101). 
The natural and interesting question in high dimensions is whether there is the "canonical boundedness" parallel to the cases $n \leq 2$. Unfortunately, when $\varphi_{1}$ is generically finite, the generic degree $\operatorname{deg}\left(\varphi_{1}\right)$ can be arbitrarily large due to the interesting example of Hacon $[\mathrm{H}$, Example 2.1] in dimension 3. Nevertheless, to the authors's knowledge, the following question remained open:

Question 1.1. Let $n \geq 3$. Does there exist a constant $N(n)>0$ so that, for all canonically fibred smooth projective $n$-folds $X$ of general type, any smooth model $F$ of an irreducible component of a general fiber of $\varphi_{1}$ has the geometric genus $p_{g}(F)<N(n)$ ?

The aim of this note is then to give the negative answer to Question 1.1. To state the main result, we first fix necessary notations. Assume $X$ is canonically of fiber type. Take a birational modification $\pi: X^{\prime} \longrightarrow$ $X$ so that $X^{\prime}$ is smooth and $\varphi_{\left|K_{X^{\prime}}\right|}$ is a morphism. From the Stein factorization of $\varphi_{\left|K_{X^{\prime}}\right|}$, one gets an induced fibration $f: X^{\prime} \longrightarrow \Gamma$ onto a lower dimensional projective variety $\Gamma$. Denote by $F$ a general fiber of $f$. Our main result is the following:

Theorem 1.2. For any set of integers $n \geq 3,0<d<n$ and $N>0$, there exists a nonsingular projective $n$-dimensional variety $X$ of general type so that $X$ is canonically fibred by d-dimensional sub-varieties $F$ with $p_{g}(F)>N$.

Theorem 1.2, implied by Examples 2.1, 2.2 and Example 2.3, clearly disproves the desired "canonical boundedness" in high dimensions. In fact, Example 2.1 does the case $d=n-2$. Example 2.2 does the cases: $1 \leq d \leq n-2$ and Example 2.3 does the case $d=n-1$.

Throughout this paper, the symbol " $X_{n, d}$ " means a projective minimal (terminal) $n$-fold which is canonically fibred by sub-varieties of dimension $d(1 \leq d<n)$.

\section{Construction of examples}

Example 2.1. The variety $X_{n, n-2}$. We consider a nonsingular projective minimal surface $S$, with $p_{g}(S)=4$ and $q(S)=0$, which was first constructed by Beauville [Bea, Proposition 3.6]. We recall that, such a surface $S$ has an involution $\sigma_{S}$ of which the fixed locus consists of 20 isolated points so that the quotient surface $T:=S /\left\langle\sigma_{S}\right\rangle$ is a quintic surface (with only 20 ordinary double points) in $\mathbb{P}^{3}$. Moreover, by the construction, the canonical system $\left|K_{S}\right|$ is base-point-free and is hence $\sigma_{S}$-invariant. Denote by $\pi: S \rightarrow T$ the corresponding double cover. Then one has $\left|K_{S}\right|=\pi^{*}\left|K_{T}\right|$ or, in other words, $\Phi_{\left|K_{S}\right|}$ factors through $\pi$ and $\Phi_{\left|K_{T}\right|}$.

Now we take any double covering $Y \rightarrow A$ from a smooth projective variety of general type to an abelian variety of dimension $d>0$ with 
the corresponding involution $\sigma_{Y}$. Define $X$ to be the quotient

$$
X_{d+2, d}:=(Y \times S) /\left\langle\sigma_{Y} \times \sigma_{S}\right\rangle .
$$

Then $X$ has terminal singularities and, by considering the natural quotient $f: X \rightarrow A \times T$, we see that $\left|K_{X}\right|=f^{*}\left|K_{A \times T}\right|$ and that $p_{g}(X)=h^{0}\left(A \times T, \omega_{A \times T}\right)$. Hence $\Phi_{\left|K_{X}\right|}$ naturally factors through the following quotient morphism:

$$
X:=(Y \times S) /\left\langle\sigma_{Y} \times \sigma_{S}\right\rangle \rightarrow S /\left\langle\sigma_{S}\right\rangle=T
$$

and $\Phi_{\left|K_{T}\right|}$. Since $n:=\operatorname{dim} X=d+2$ and $\operatorname{dim} \Phi_{\left|K_{T}\right|}(T)=2$, we see that $X$ is canonically fibred by varieties (isomorphic to $Y$ ) of dimension $d$. It is clear that $p_{g}(Y)$ can be arbitrary large.

From the construction of $X_{n, n-2}$, we see that the induced fibration from $\Phi_{\left|K_{X_{n, n-2} \mid}\right|}$ is iso-trivial with the general fiber a fixed double cover of the given abelian $d$-fold $A$.

Example 2.2. Varieties $X_{n, d}$ with $1 \leq d \leq n-2$. Let $A_{i}$ be a simple principally polarized abelian variety of dimension $d_{i}>0$ with a principal polarization $\Theta_{i}$ for $i=1,2$. The existence of such pairs $\left(A_{i}, \Theta_{i}\right)$ is well-known. Let $\rho_{i}: F_{i} \longrightarrow A_{i}$ be the double cover ramified over a general member of $\left|2 \Theta_{i}\right|$. Hence $F_{i}$ is a smooth variety of general type. Write

$$
\rho_{i *} \omega_{F_{i}}=\mathscr{O}_{F_{i}} \oplus \Theta_{i}
$$

and denote by $\sigma_{i}$ the involution corresponding to $\rho_{i}$ for each $i=1,2$. Clearly we have $p_{g}\left(F_{i}\right)=2$ for each $i$.

Let $S$ be a smooth projective variety of dimension $d_{3} \geq 1$ with $p_{g}(S)=0$. Take a very ample line bundle $L$ on $S$ such that $K_{S}+L$ is also very ample. Pick a smooth divisor $D \in|2 L|$. Let $\rho_{3}: T \rightarrow S$ be a double cover of $S$ ramified in $D$. Then $T$ is a smooth projective variety of general type and we have

$$
\rho_{3 *} \omega_{T}=\omega_{S} \oplus\left(\omega_{S} \otimes L\right) .
$$

Denote by $\sigma_{3}$ the involution corresponding to $\rho_{3}$.

Step 1. We consider the product $F_{1} \times F_{2} \times T$ and the involution $\sigma=\sigma_{1} \times \sigma_{2} \times \sigma_{3}$. Define $X$ to be the quotient $\left(F_{1} \times F_{2} \times T\right) /\langle\sigma\rangle$. Then $X$ is a minimal projective variety with terminal singularities of Cartier index 2 and $K_{X}^{3}>0$.

Let $\rho: X \rightarrow Z:=A_{1} \times A_{2} \times S$ be the natural morphism which is clearly a $(\mathbf{Z} / 2 \mathbf{Z} \times \mathbf{Z} / 2 \mathbf{Z})$-covering. Moreover, by equations (11) and (2), we have

$$
\begin{aligned}
\rho_{*} \omega_{X}= & \left(\mathscr{O}_{A_{1}} \otimes \mathscr{O}_{A_{2}} \otimes \omega_{S}\right) \oplus\left(\Theta_{1} \otimes \Theta_{2} \otimes \omega_{S}\right) \\
& \oplus\left(\Theta_{1} \otimes \mathscr{O}_{A_{2}} \otimes\left(\omega_{S} \otimes L\right)\right) \oplus\left(\mathscr{O}_{A_{1}} \otimes \Theta_{2} \otimes\left(\omega_{S} \otimes L\right)\right),
\end{aligned}
$$

where $\mathscr{O}_{A_{1}} \otimes \mathscr{O}_{A_{2}} \otimes \omega_{S}:=\iota_{1}^{*} \mathscr{O}_{A_{1}} \otimes \iota_{2}^{*} \mathscr{O}_{A_{2}} \otimes \iota_{3}^{*} \omega_{S}$ and $\iota_{i}$ is the projection from $A_{1} \times A_{2} \times S$ onto the $i$-th factor for $i=1,2,3$. 
In particular, we have

$$
\begin{aligned}
H^{0}\left(X, \omega_{X}\right) \cong & H^{0}\left(Z, \mathscr{O}_{A_{1}} \otimes \mathscr{O}_{A_{2}} \otimes \omega_{S}\right) \oplus H^{0}\left(Z, \Theta_{1} \otimes \Theta_{2} \otimes \omega_{S}\right) \\
& \oplus H^{0}\left(Z, \Theta_{1} \otimes \mathscr{O}_{A_{2}} \otimes\left(\omega_{S} \otimes L\right)\right) \oplus H^{0}\left(Z, \mathscr{O}_{A_{1}} \otimes \Theta_{2} \otimes\left(\omega_{S} \otimes L\right)\right) .
\end{aligned}
$$

Besides, we have the following commutative diagram:

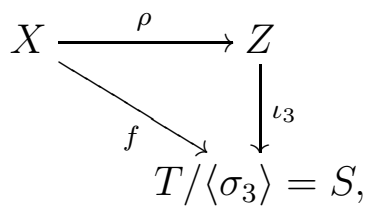

where $f:=\iota_{3} \circ \rho$. By (3) , we have

$$
f_{*} \omega_{X} \simeq \omega_{S} \oplus \omega_{S} \oplus\left(\omega_{S} \otimes L\right) \oplus\left(\omega_{S} \otimes L\right) .
$$

For the general point $t \in S$, denote by $X_{t}$ the general fiber of $f$ over $t$. By direct calculation, one has $X_{t} \simeq F_{1} \times F_{2}$ and, clearly, $p_{g}\left(X_{t}\right)=$ $p_{g}\left(F_{1}\right) \cdot p_{g}\left(F_{2}\right)=4$. Then the restriction map

$$
H^{0}\left(X, \omega_{X}\right) \rightarrow H^{0}\left(X_{t}, \omega_{X_{t}}\right)
$$

has the image dimension $=2$ since $h^{0}\left(A_{i}, \Theta_{i}\right)=1$ for $i=1,2$ by assumption. Here we note that $f_{*} \omega_{X}$ has rank 4. Hence, the image $\overline{\Phi_{\left|K_{X}\right|}(X)}$ is birational to a $\mathbf{P}^{1}$-bundle $V$ over $S$. Thus we have the following diagram:

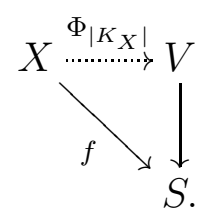

Denote by $F$ an irreducible component of a general fiber of $\Phi_{\left|K_{X}\right|}$. As we have already seen, $F$ is a sub-canonical divisor of $K_{X_{t}}=K_{F_{1} \times F_{2}}$. In fact, $F$ moves in a sub-pencil of $\left|K_{X_{t}}\right|$.

Step 2. We note that the natural morphism $\alpha: X \rightarrow A_{1} \times A_{2}$ is the Albanese morphism of $X$. We take $P=P_{1} \otimes P_{2}$, a general $m$-torsion line bundle of $X$ and let $\pi_{m}: X_{m} \longrightarrow X$ be the étale $m$-cover of $X$ induced by $P$. Then we have $\pi_{m *} \omega_{X_{m}}=\oplus_{i=0}^{m}\left(\omega_{X} \otimes P^{\otimes i}\right)$. Since both $P_{1}$ and $P_{2}$ are of order $m$, by (3), we have $H^{0}\left(X_{m}, \omega_{X_{m}}\right) \cong H^{0}\left(X, \omega_{X}\right)$. Thus, we have the following commutative diagram:

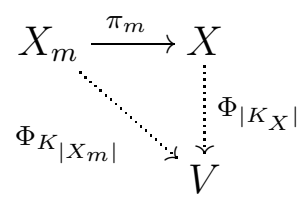

Moreover, since $\alpha(F)$ generates $A_{1} \times A_{2}$, we can choose a very general $m$-torsion $P$ such that $\left.P\right|_{F}$ is also a $m$-torsion (noting that $F$ moves in an algebraic family on $X_{t}$ ). Thus, over $F$, the irreducible component $F_{m}$ of the general fiber of $\Phi_{\left|K_{X_{m}}\right|}$ is simply an étale $m$-cover of $F$. 
Take a desingularization $\varepsilon: F^{\prime} \rightarrow F$. Then the induced étale cover $F_{m}^{\prime}$ of $F^{\prime}$ is a desingularization of $F_{m}$. Consider the induced generically finite morphism $\beta:=\left.\alpha\right|_{F} \circ \varepsilon: F^{\prime} \rightarrow A_{1} \times A_{2}$. Since $F^{\prime}$ is of general type and both $A_{1}$ and $A_{2}$ are simple, we have $H^{0}\left(F^{\prime}, \omega_{F^{\prime}} \otimes \beta^{*} Q\right) \neq 0$, for any $Q \in \operatorname{Pic}^{0}\left(A_{1} \times A_{2}\right)$ (see [CDJ, Corollary 3.5]). Therefore, $\lim _{m \rightarrow \infty} p_{g}\left(F_{m}^{\prime}\right) \rightarrow+\infty$.

We see that $X_{m}$ is minimal projective with terminal singularities and that $X_{m}$ is canonically fibred by varieties which are algebraically equivalent to $F_{m}$. Moreover, $\operatorname{dim} X_{m}=d_{1}+d_{2}+d_{3} \geq 3$ and $\operatorname{dim} F_{m}=$ $d_{1}+d_{2}-1$. Hence, choosing appropriate $d_{1}, d_{2}$, and $d_{3}$, $\operatorname{dim} F_{m}$ can be any integer between 1 and $\operatorname{dim} X_{m}-2$. We set $X_{n, d}^{<m>}:=X_{m}$ where $n=d_{1}+d_{2}+d_{3}$ and $d=d_{1}+d_{2}-1$.

Clearly, as $m$ goes to infinity, our variety $X_{n, d}^{<m>}$ is a minimal $n$ fold of general type which is canonically fibred by sub-varieties $F_{m}$ of dimension $d$ with $1 \leq d \leq n-2$ and, furthermore, the smooth model of $F_{m}$ has arbitrarily large geometric genus.

Example 2.3. The variety $X_{n, n-1}$. We consider a variant of ChenHacon's example [CH1, §4] where a threefold of general type, with maximal Albanese dimension and with $p_{g}=1$, was constructed.

Step 1. To be precise, let $\left(A_{i}, \Theta_{i}\right)$ be simple principal polarized abelian varieites for $i=1,2,3$. Let $\rho_{i}: F_{i} \rightarrow A_{i}$ be the double covering ramified over a general element of $\left|2 \Theta_{i}\right|$, with the induced involution $\sigma_{i}$, for each $i$. Choose $P_{i} \in \operatorname{Pic}^{0}\left(A_{i}\right)$ of order 2 , we consider the induced étale covers $F_{i}^{\prime} \rightarrow F_{i}$, with the induced involution $\iota_{i}$. The involution $\sigma_{i}$ on $F_{i}$ pulls back to an involution $\sigma_{i}^{\prime}$ on $F_{i}^{\prime}$.

We then define $X$ to be the quotient of $F_{1}^{\prime} \times F_{2}^{\prime} \times F_{3}^{\prime}$ by the group of automorphisms generated by $\operatorname{id}_{F_{1}^{\prime}} \times \sigma_{2}^{\prime} \times \iota_{3}, \iota_{1} \times \operatorname{id}_{F_{2}^{\prime}} \times \sigma_{3}^{\prime}, \sigma_{1}^{\prime} \times \iota_{2} \times \operatorname{id}_{F_{3}^{\prime}}$, $\sigma_{1}^{\prime} \times \sigma_{2}^{\prime} \times \sigma_{3}^{\prime}$.

One can check that $X$ is minimal projective of general type with terminal singularities of Cartier index 2. Moreover, we have a $(\mathbf{Z} / 2 \mathbf{Z} \times$ $\mathbf{Z} / 2 \mathbf{Z}$ )-cover:

$$
\rho: X \rightarrow A:=A_{1} \times A_{2} \times A_{3},
$$

and by analyzing the action of the Galois group, we have

$$
\begin{aligned}
\rho_{*} \omega_{X}= & \mathscr{O}_{A} \oplus\left(\Theta_{1} \otimes\left(\Theta_{2} \otimes P_{2}\right) \otimes P_{3}\right) \oplus\left(\left(\Theta_{1} \otimes P_{1}\right) \otimes P_{2} \otimes \Theta_{3}\right) \\
& \oplus\left(P_{1} \otimes \Theta_{2} \otimes\left(\Theta_{3} \otimes P_{3}\right)\right) .
\end{aligned}
$$

We note that $\rho$ is the Albanese morphism of $X$. Modulo one more étale cover of $A_{1}$ to annihilate $P_{1}$ upstairs, we may and do assume that $P_{1}=\mathscr{O}_{A_{1}}$ is trivial. In other words, we have got such a variety $X$ that $\rho_{*} \omega_{X}$ has exactly two summands contributing to its global sections, which are $\mathscr{O}_{A}$ and $\mathscr{O}_{A_{1}} \otimes \Theta_{2} \otimes\left(\Theta_{3} \otimes P_{3}\right)$ by (4). Hence we have $\operatorname{dim} H^{0}\left(X, \omega_{X}\right)=2$. We denote $\operatorname{dim} X=n \geq 3$, then automatically $X$ is canonically fibred by varieties of dimension $n-1$. 
Step 2. We consider the the natural morphism $f: X \rightarrow A_{1}$. Then $f_{*} \omega_{X}=\mathscr{O}_{A_{1}} \oplus \mathscr{O}_{A_{1}}$. Hence, the restriction of the canonical pencil $\left|K_{X}\right|$ to a general fiber of $f$ is still a pencil. Then, up to birational equivalence, $\Phi_{\left|K_{X}\right|}$ does not factor through $f$. Let $F$ be any irreducible component of the general fiber of $\Phi_{\left|K_{X}\right|}$. Then $\left.f\right|_{F}: F \rightarrow A_{1}$ is surjective. In particular, for any integer $m>1$, there exists a torsion line bundle $P_{m}$ on $A_{1}$ such that $\left.f^{*} P_{m}\right|_{F}$ is still a $m$-torsion.

We then take a desingularization $\varepsilon: F^{\prime} \rightarrow F$. Considering the induced generically finite morphism: $\beta:=\left.\rho\right|_{F} \circ \varepsilon: F^{\prime} \rightarrow A$, since $A$ has only 3 simple factors and $\beta$ is not surjective, we have $H^{0}\left(F^{\prime}, \omega_{F^{\prime}} \otimes\right.$ $\left.\beta^{*} Q\right) \neq 0$, for any $Q \in \operatorname{Pic}^{0}(A)$ (see [CDJ, Proposition 4.3]).

Finally let $\pi_{m}: X_{m} \rightarrow X$ be the étale $m$-cover induced by $f^{*} P_{m}$. By (44), we can easily check that $H^{0}\left(X_{m}, \omega_{X_{m}}\right) \cong H^{0}\left(X, \omega_{X}\right)$ and $\omega_{X_{m}}=$ $\pi_{m}^{*} \omega_{X}$. Thus, we have the commutative diagram:

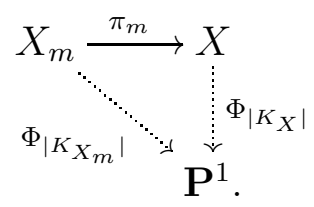

Clearly $\left|K_{X_{m}}\right|$ is composed of a pencil of $(n-1)$-dimensional varieties. Any irreducible element $F_{m}$ of the general fiber of $\Phi_{\left|K_{X_{m}}\right|}$ is then an étale $m$-cover of $F$ induced by $\left.f^{*} P_{m}\right|_{F}$. Let $F_{m}^{\prime}$ be the corresponding étale cover of $F^{\prime}$, then $F_{m}^{\prime}$ is a desingularization of $F^{\prime}$ and simple calculations tell us that $\lim _{m \rightarrow \infty} p_{g}\left(F_{m}^{\prime}\right) \rightarrow+\infty$. Set $X_{n, n-1}^{<m>}$, which is what we want to realize.

Clearly, as $m$ goes to infinity, $X_{n, n-1}^{<m>}$ is a projective minimal (terminal) $n$-fold and is canonically fibred by $(n-1)$-folds $F_{m}$. Furthermore, a smooth model of $F_{m}$ has arbitrarily large geometric genus.

Remark 2.4. Though the "canonical boundedness" does not hold in dimension $\geq 3$ by Theorem 1.2, we still have boundedness results in special cases. For example, if we consider canonically fibred Gorenstein minimal 3-folds of general type, Chen and Hacon [CH2, Theorem 1.1] showed that the fiber invariants of $\Phi_{\left|K_{X}\right|}$ are upper bounded, and some interesting examples with large fibre invariants were found by Chen-Cui in $[\mathrm{CC}]$.

It is then natural to ask the following questions:

Question 2.5. Let $m>1$ and $n \geq 3$ be two integers. Considering all those projective minimal $n$-folds of general type which are $m$ canonically fibred, are the birational invariants of fibers of $\Phi_{|m K|}$ universally bounded from above?

Question 2.6. Let $Y$ be a projective Gorenstein minimal $n$-fold ( $n \geq$ 4) of general type with $p_{g}(Y) \geq 2$. 
(1) Assume that $\varphi_{1}$ is generically finite. Is $\operatorname{deg}\left(\varphi_{1}\right)$ bounded from above?

(2) Assume that $\varphi_{1}$ is of fiber type. Is the geometric genus of any smooth model of any irreducible component in the general fiber of $\varphi_{1}$ bounded from above?

(3) Is there an effective inequality of the form $K_{Y}^{n} \leq c(n) p_{g}(Y)$ where $c(n)$ is a constant depending only on $n$ ?

\section{REFERENCES}

[BHPV] W. Barth, K. Hulek, C. Peters, A. Van de Ven, Compact complex surfaces. Second edition. Ergebnisse der Mathematik und ihrer Grenzgebiete. 3. Folge. A Series of Modern Surveys in Mathematics, 4. Springer-Verlag, Berlin, 2004. xii+436 pp.

[Bea] A. Beauville, L'application canonique pour les surfaces de type général, Invent. Math. 55 (1979), no. 2, 121-140.

[CDJ] J. A. Chen, O. Debarre, and Z. Jiang, Varieties with vanishing holomorphic Euler characteristic 691 (2014), 203-227.

[CH1] J. A. Chen, and C. D. Hacon, On the irregularity of the image of the Iitaka fibration, Comm. Algebra 32 (2004), 203-215.

$[\mathrm{CC}] \quad$ M. Chen, and A. Cui, On canonically fibred algebraic 3-folds-Some new examples, Pure and Applied Mathematics Quarterly 7 (2011), 1105-1128.

[CH2] M. Chen, and C. D. Hacon, On the geography of Gorenstein minimal 3-folds of general type, Asian J. Math. 10 (2006), no.4, 757-763.

[H] C. D. Hacon, On the degree of the canonical maps of 3-folds, Proc. Japan Acad. Ser. A Math. Sci. 80 (2004), no.8, 166-167.

School of Mathematical Sciences \& LMNS, Fudan University, Shanghai 200433, China

E-mail address: mchen@fudan.edu.cn

Département de Mathématiques, Bâtiment 425, Université Paris-Sud, F-91405 Orsay, France

E-mail address: zhi.jiang@math.u-psud.fr 